\title{
Development and Characterisation of an Easily Configurable Range Imaging System
}

\author{
Adrian P. P. Jongenelen, Dale A. Carnegie \\ School of Engineering and Computer Science \\ Victoria University of Wellington \\ Wellington, New Zealand \\ adrian.jongenelen@vuw.ac.nz
}

\author{
Andrew D. Payne, Adrian A. Dorrington \\ Department of Engineering \\ University of Waikato \\ Hamilton, New Zealand
}

\begin{abstract}
Range imaging is becoming a popular tool for many applications, with several commercial variants now available. These systems find numerous real world applications such as interactive gaming and the automotive industry.

This paper describes the development of a range imaging system employing the PMD-19k sensor from PMD Technologies. One specific advantage of our system is that it is extremely customisable in terms of modulation patterns to act as a platform for further research into time-of-flight range imaging. Experimental results are presented giving an indication of the precision and accuracy of the system, and how modifying certain operating parameters can improve system performance.
\end{abstract}

Keywords- range imaging, FPGA, image sensor, time-offlight, $3 D$ camera.

\section{INTRODUCTION}

Recent years have seen a great deal of advancement in the area of full-field range imaging using the indirect Time-ofFlight (TOF) technique $[1,2,3]$. The goal is to capture an image of a scene where each pixel of the image encodes both distance and intensity information. A number of systems are available for purchase $[4,5,6,7]$ that are complete with custom camera sensor IC and integrated illumination. These are useful for many applications such as machine vision, automotive safety, entertainment and robotics.

While these commercial systems do allow some configurability of the modulation signals, they are limited to a choice of fixed frequencies and modulation schemes. For our research we wish to have much finer control over the operating parameters of the range imaging system. We aim to construct a range imaging system where the configuration can be easily changed through software and the modulation signals driven by a user configurable FPGA. This allows for a great degree of flexibility providing an excellent platform for further research and a greater understanding of the effects of modulation schemes.

In Section II the underlying concepts of range imaging using indirect time-of-flight techniques are explained including a description of factors that affect measurement precision and accuracy. Section III explains in detail each of the hardware components that comprise the range imaging system constructed by the authors.
In Section IV we present the results of a series of characterising tests performed on the system and discuss the performance in terms of precision and accuracy, with a summary and conclusion in Section V.

\section{INDIRECT TIME-OF-FLIGHT RANGING OVERVIEW}

\section{A. System Operation}

The primary advantage of the indirect TOF method for acquiring range data compared with other methods such as point scanning, structured light and stereo vision is the ability of the system to find range for every pixel of the image sensor simultaneously. The system has no moving parts and the algorithm for determining range can be applied independently per pixel and can be efficiently implemented in hardware.

The scene is illuminated by a light source modulated at a high frequency, $f_{M}$, typically between 10 and $100 \mathrm{MHz}$. The light is reflected from the scene and imaged by a sensor with an electronic shutter that is also modulated at the same frequency. The effective signal integrated by each pixel is the shutter signal mixed with returned light from the scene. This returned light has a modulation envelope phase delay due to the time taken for the light to travel to and from the objects in the scene. As a result, the pixel intensity is proportional to the modulation envelope phase delay of the returned light, and hence distance.

Using only one image frame it is impossible to know if the intensity of the pixel is a function of the phase delay or simply that the returned light is of lower intensity due to poor reflectance of the target object, changes in ambient light levels, or from the $1 / d^{2}$ relationship between light intensity over distance. To overcome this it is typical to take a number of frames, $N$ where the initial phase offset between the transmitted light and sensor modulation is stepped by $2 \pi / N$ radians between frames.

\section{B. Phase Determination}

For sinusoidal modulation the pixel intensity across consecutive raw data frames also represents a sinusoid, sampled in time. The phase delay of the returned light can therefore be found by calculating the phase of this sampled sinusoid using the formula 


$$
\theta=\arctan \left(\frac{\sum_{i=0}^{N-1} I_{i} \sin \left(\frac{2 \pi i}{N}\right)}{\sum_{i=0}^{N-1} I_{i} \cos \left(\frac{2 \pi i}{N}\right)}\right)
$$

where $I_{i}$ are the pixel values from each of the $N$ raw data frames per cycle.

In practice however it is generally simpler to drive the modulation with a digital switching circuit giving modulation of a square waveform. In the case of perfect square wave modulation the theoretical pixel intensity can be modelled by a triangular wave, although in practice it is more sinusoidal due to bandwidth limitations of the hardware [8]. Nevertheless, the same formula for phase determination is still used.

\section{Distance Determination}

The distance of the object to the imaging system can then be calculated as half (because of the return trip) the fractional wavelength of the modulation signal by

$$
d=\frac{c}{2 f_{M}}\left(\frac{\theta}{2 \pi}+k\right)=d_{u}\left(\frac{\theta}{2 \pi}+k\right)
$$

where $k$ is an integer, $f_{M}$ is the modulation frequency and $c$ is the speed of light. The integer $k$ represents the problem where it is possible for the phase of the returned light to be delayed by an extra multiple of $2 \pi$ radians. This gives rise to an ambiguity in the distance measurement and the concept of the maximum unambiguous range, $d_{u}$. Methods for extending the unambiguous range include using a relatively low modulation frequency or taking a combination of multiple modulation frequency captures and calculating the result as the lowest common multiple [9].

\section{Precision}

Precision in this case is a measure of the repeatability of the results across consecutive range image frames. This can be measured by taking a number of range images of a static scene and calculating the standard deviation of the distance. The standard deviation of the phase measurement, $\sigma_{P}$, is inversely proportional to the square root of the signal-to-noise ratio (SNR) of the received pixel intensities by the formula [10]

$$
\sigma_{P}=\frac{1}{m \sqrt{S N R}}
$$

where $m$ is the modulation index describing the relationship between the maximum and minimum levels of the modulation waveform, typically between 0.3 and 0.7. The SNR encompasses noise from all sources and is comprised of a number of factors which include:

- intensity of reflected light,
- intensity of ambient light,

- electrical noise,

- quantisation errors,

- $\quad$ sensor integration time.

In most applications outside the lab environment ambient light intensity can not be controlled. While it is possible to influence the intensity of the reflected light by increasing the output power of the illumination source there is still a large amount of variation dependant on the reflectance properties and distance to objects in the scene. Electrical noise and quantisation errors can be addressed to some extent during the design of the system, but thereafter they are outside the control of the user. This leaves sensor integration time as the predominant control for phase precision.

Range precision, $\sigma_{R}$, can be estimated by converting phase measurement uncertainty to distance uncertainty with

$$
\sigma_{R}=\sigma_{P} \frac{c}{4 \pi f_{M}}
$$

It follows that range precision is inversely proportional to modulation frequency, and therefore can also be affected by the user configurable modulation frequency selection.

\section{E. Accuracy}

Accuracy is a measure of how closely the range data represents the true distance to the object. In addition to the problem of accurately measuring objects beyond the maximum unambiguous range there are also systematic errors introduced in the phase measurement due to aliased harmonics in the pixel intensity waveform [11].

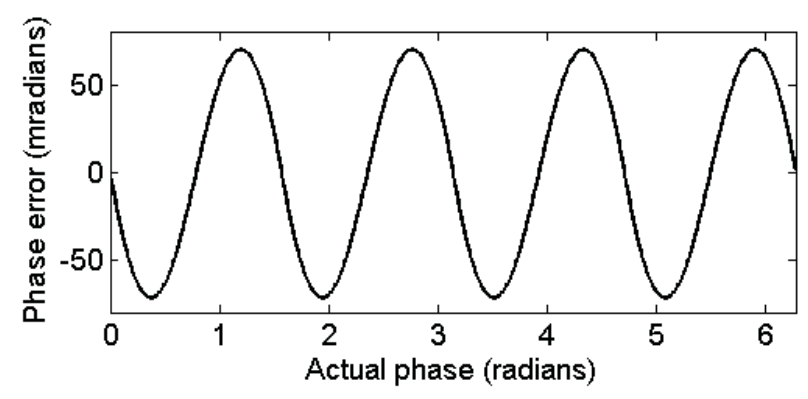

Figure 1. Simulated phase error versus actual phase offset for a triangular pixel intensity waveform sampled at 4 frames per cycle.

Fig. 1 shows the simulated phase error for $N=4$ using a theoretical worst case triangule wave pixel intensity waveform, where the modulation signal is assumed to be perfectly square. Through careful selection of $N$, and hence the Nyquist frequency, it is possible to reduce the number of harmonics that are aliased onto the fundamental frequency during sampling. Fig. 2 shows the simulated RMS phase error for three to ten frames. Commonly four frames per cycle are used in the industry due to the simplicity of generating the phase offsets and processing the image sequence. Although the results 
shown are from simulation only, and actual results will vary with system implementation due to varying harmonic content and system bandwidth limits, it is evident there may be value to be had in terms of phase accuracy by designing a system that is flexible in this respect.

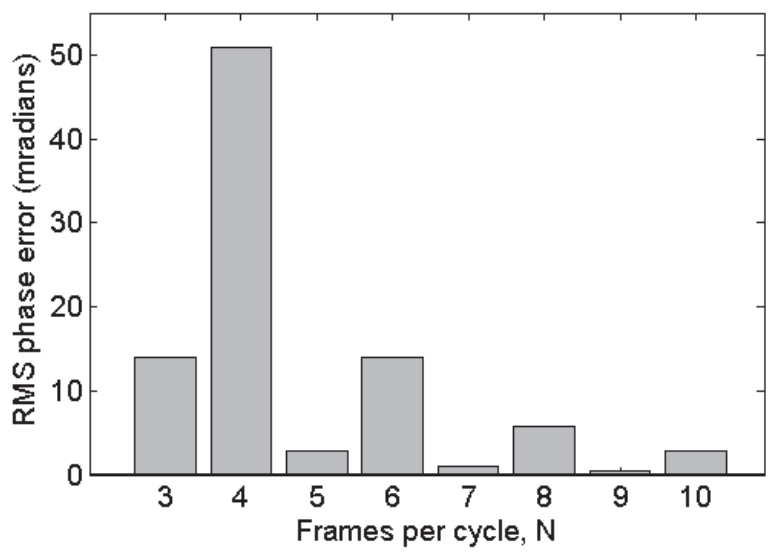

Figure 2. Simulated RMS phase error for frames per cycle of 3 to 10 for square wave modulation.

\section{HARDWARE DESIGN}

The complete ranging system comprises a number of interconnected components as shown in Fig. 3. Optical signals are shown as black arrows, electronic control signals as gray arrows and the path of image frame data shown as white arrows. The following sections describe each of these components in more detail.

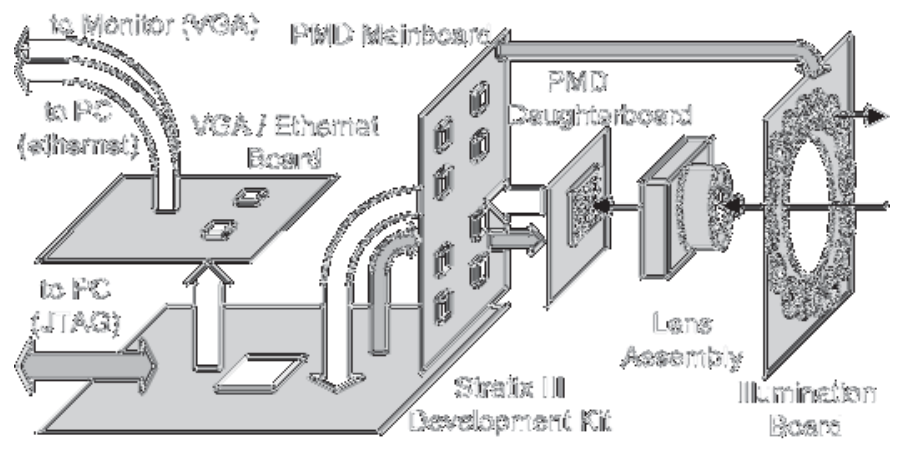

Figure 3. Interaction between the hardware components of the ranging system. Optical signals are represented as black arrows, modulation and control signals as grey arrows, and the image data path as white arrows.

\section{A. Stratix III Development Kit}

The Stratix III Development Kit hosts an Altera Stratix III EP3SL150F1152 FPGA [12]. This has been selected primarily for the easily reconfigurable phase-locked-loop (PLL) resources. Each PLL can provide up to 10 phase synchronous output signals of frequencies up to $700 \mathrm{MHz}$.

The main advantage of these PLLs is the ability to reconfigure the phase, frequency and duty cycle of each output channel in real-time. This is an especially useful feature for testing the system under a large number of operating conditions. The ability to change the modulation signal both between frames as well as during the sensor integration time provides an excellent platform for further research into various range imaging modulation schemes.

The PLL has a voltage controlled oscillator (VCO) with a frequency, $f_{V C O}$ set by

$$
f_{V C O}=\frac{f_{I N} c_{m}}{c_{n}}
$$

where $f_{I N}$ is an input frequency of $50 \mathrm{MHz}$ from the onboard crystal oscillator, and $c_{m}$ and $c_{n}$ are configurable integers. The PLL output frequency, $f_{\text {OUT }}$ is determined by

$$
f_{\text {OUT }}=\frac{f_{V C O}}{c_{H I}+c_{L O}}
$$

where $c_{H I}$ and $c_{L O}$ are configurable integers. The duty cycle can therefore be controlled by the ratio between $c_{H I}$ and $c_{L O}$. The output phase is set by incrementing the phase in discrete steps, with each step giving a phase shift in radians of

$$
\phi_{\text {step }}=\frac{2 \pi}{8\left(c_{H I}+c_{L O}\right)}
$$

For example, an output frequency of $40 \mathrm{MHz}$ can be achieved using $c_{m}=24, c_{n}=1, c_{H I}=15$ and $c_{L O}=15$, giving 240 discrete and equally spaced phase steps before repeating.

In addition to driving the modulation signals the FPGA is also responsible for:

- $\quad$ timing and control signals for retrieving image data from the PMD sensor;

- handling the JTAG interface to a PC to process user commands and configure operating parameters;

- processing the raw image data and calculating range images;

- temporarily storing both raw and processed data;

- timing and control of signals for displaying range image data on a VGA monitor;

- handling an Ethernet connection to a PC for transferring image data for long term storage.

The interface to the PMD sensor and the VGA monitor, and the real-time image processing and storage are programmed using VHDL. For the JTAG interface and Ethernet connection a Nios II processor core is used in the design and programmed in $\mathrm{C}$. 


\section{B. Illumination Board}

Active illumination of the scene is provided by 16 laser diodes mounted in a circular pattern on the illumination board. There are two independent banks of eight diodes each: one set of red $658 \mathrm{~nm}$ wavelength the other with near infra-red (NIR) $808 \mathrm{~nm}$ wavelength. These are independently driven in a controlled current configuration giving a total continuous wave optical output power of $800 \mathrm{~mW}$. The modulation signals provided by the Stratix III FPGA are provided through the PMD Mainboard using low voltage differential signalling (LVDS).

The illumination board also features a Microchip Technology PIC16F684 microcontroller whose purpose, in combination with a pair of Analog Devices AD5311 digital-toanalogue converters, is to control the current through the laser diodes. To prevent damage to the diodes, the microcontroller first checks that the duty cycle of the modulation signal is not too high or too low and then begins a warm-up phase where the current ramps up in a slow, predefined manner up to the maximum operating current. If the modulation stops the microcontroller automatically shuts off the current and returns to the warm-up phase.

\section{PMD Daughterboard}

A PMDTechnologies PMD19k-2 image sensor [13] is used. It features a $160 \times 120$ array of pixels grouped into four independently modulated blocks of $40 \times 120$ pixels, with each block representing a capacitive load of $250 \mathrm{pF}$. The PMD Daughterboard hosts the image sensor and contains only a bare minimum of passive components required to set various voltage reference levels and filter the power supply. All modulation, analogue video outputs and control signals are routed via a connector to the Mainboard. This allows design changes to be made to the Mainboard without directly affecting the image sensor.

\section{PMD Mainboard}

The PMD Mainboard hosts all the electronics for interfacing the FPGA to the image sensor and illumination board. This includes:

- conversion between LVDS signalling with the FPGA and single ended CMOS signalling with the image sensor;

- analogue-to-digital conversion of the image sensor video output streams;

- high-speed drive of the high-capacitance sensor modulation inputs;

- LVDS buffering for relaying the modulation signal to the illumination board.

The large capacitive load of the sensor modulation blocks are driven using a pair of Intersil EL7158 ultra-high current pin driver ICs. The Mainboard also features a 160 pin high-speed mezzanine connector for high-bandwidth communication with the Stratix III Development Kit.

\section{E. Lens Assembly}

Mounted on the PMD Daughterboard is a cage plate designed to hold a C-Mount lens. For this project a Goyo Optical $16 \mathrm{~mm}$ focal length lens with manual focus and iris is fitted. This gives a field-of-view (Horizontal $\times$ Vertical) of $22.2^{\circ} \times 16.5^{\circ}$.

The lens has a $27 \mathrm{~mm}$ filter thread to which a variety of optical filters can be attached. A LP645 filter from Midwest Optical Systems is used which passes over $90 \%$ of the reflected light of the red and NIR laser diodes while excluding shorter wavelength visible background light that the image sensor would otherwise detect.

\section{F. VGA / Ethernet Board}

Raw and processed image frames are temporarily stored in RAM on the Stratix III Development Kit. Longer term storage is possible through an Ethernet link to a PC where the image frames can be saved to a file. This is handled by a DAVICOM DM9000A Ethernet Controller mounted on the VGA / Ethernet board.

In addition frames are displayed in real-time by a standard $640 \times 480$ resolution VGA monitor. The VGA / Ethernet board hosts an Analog Devices ADV7123 3-channel digital-toanalogue converter for driving the analogue red, green, and blue signals to the VGA monitor.

This board also has a group of spare connections to the FPGA for debugging or incorporating additional hardware at a later date.

\section{SYSTEM CHARACTERISATION}

To test the relationship between the modulation frequency and precision a static scene is set up with a flat panel positioned $2.30 \mathrm{~m}$ from the image sensor. A 3D reconstruction of the scene created from range data is shown in Fig. 4 where the grey colour scale represents distance in $\mathrm{mm}$.

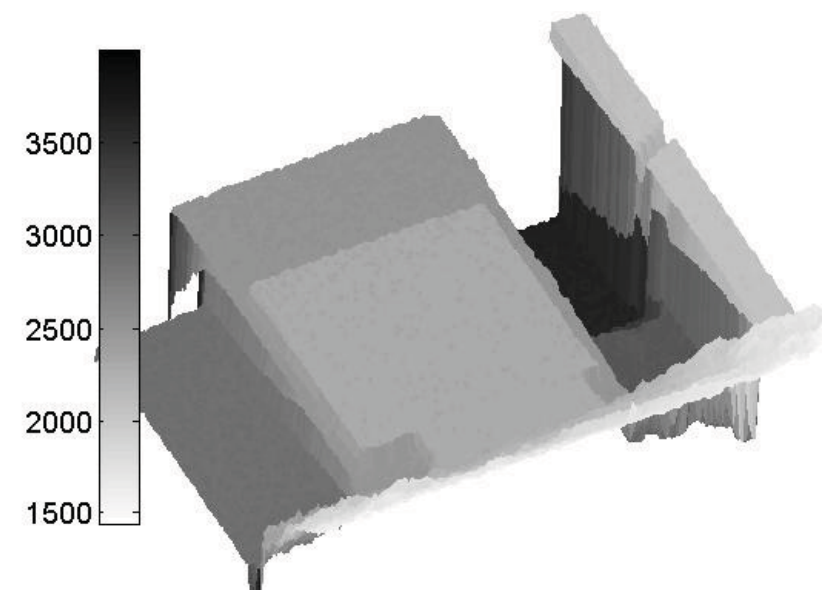

Figure 4. 3D reconstruction of the test scene for characterisation. The grey colour scale represents distance in $\mathrm{mm}$.

The precision is measured by taking the standard deviation of each pixel over 40 range measurements and averaged for an 
$8 \times 8$ group of pixels located on the panel. Frames per cycle, $N$, is fixed at 4 , (a value commonly used by other range imaging systems), and the total integration time for each range image cycle is set to $200 \mathrm{~ms}$, providing a range image frame rate of 5 fps.

Fig. 5 shows a plot of the phase measurement precision (with the scale on the left axis), and corresponding range measurement precision (right axis) for various modulation frequencies. It can be seen that phase precision is approximately constant at 4 milliradians for modulation frequencies up to $40 \mathrm{MHz}$, at which point phase precision becomes progressively worse due to bandwidth limitations of the electronics. Measured distance is inversely proportional to modulation frequency, therefore in the region where phase precision is constant it can be seen that distance measurement precision is improving as the frequency is increased, with a best case precision of approximately $3.5 \mathrm{~mm}$ at $36 \mathrm{MHz}$ modulation.

Another measure of performance is the accuracy of the measured distance versus the actual distance. Using the same scene, multiple captures are taken with modulation frequencies of 10, 20 and $40 \mathrm{MHz}$. Rather than moving the target object which might change other parameters such as the intensity of the returned light, movement of the object is synthesised by applying small incremental adjustments to the phase of the sensor modulation signal. One calibration is performed to fix the first capture to an error of $0 \mathrm{~mm}$.

Fig. 6 shows a plot of the mean measured distance error versus synthesised distance offset for the three modulation frequencies. The $\mathrm{x}$-axis shows synthesised distance offset up to $3.75 \mathrm{~m}$, which is the maximum unambiguous range using a modulation frequency of $40 \mathrm{MHz}$. The error is cyclic, with four periods present for the $40 \mathrm{MHz}$ result. For 20 and $10 \mathrm{MHz}$ modulation the maximum unambiguous range extends to 7.5 and $15 \mathrm{~m}$ respectively, hence why on this scale only two and one such periods are shown.

As mentioned in Section 2, with square wave modulation the resultant pixel intensity is inherently a triangular wave, where for $N=4$ the odd order harmonics interfere with the fundamental frequency. The $3^{\text {rd }}$ and $5^{\text {th }}$ order harmonics typically have the largest magnitude and are the cause for the large cyclic error. The error present in the $10 \mathrm{MHz}$ result is large for two reasons. Firstly, even if the raw phase errors were the same for all modulation frequencies, the resultant distance error would be larger at the lower frequency due to the inverse relationship between modulation frequency and measured distance. Secondly, as the modulation frequency is decreased the driving electronics produce a waveform which more closely resembles a square wave. This has the effect of more faithfully producing the triangular pixel intensity waveform, and consequently increasing the magnitude of odd order harmonics.

The synthesised distance error measurements were repeated, this time keeping the modulation frequency constant at $40 \mathrm{MHz}$ while changing $N$ from three to ten. The total integration time per range measurement was also kept constant at $200 \mathrm{~ms}$. The bar plot of Fig. 7 shows the resulting RMS phase error for each capture.

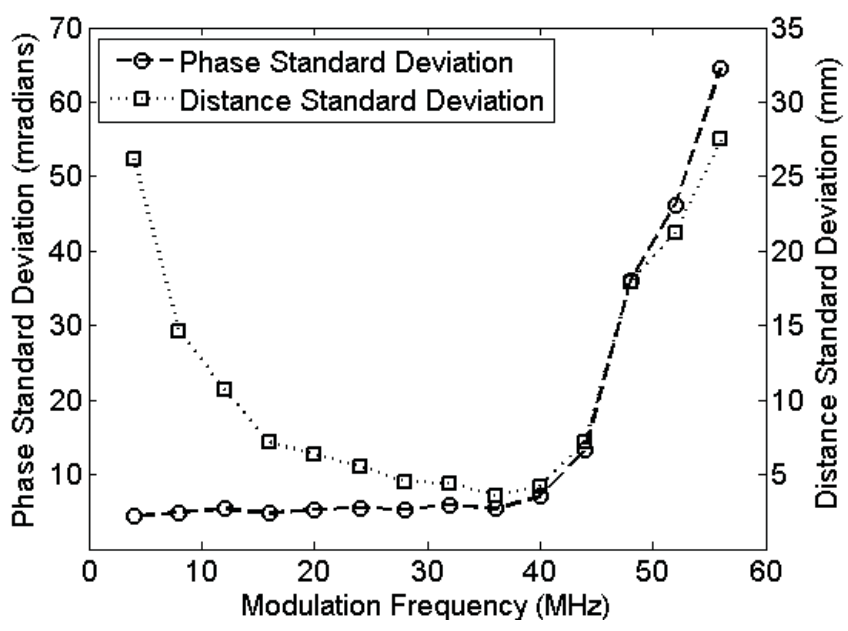

Figure 5. Modulation frequency versus phase and distance standard deviation for a $200 \mathrm{~ms}$ capture with 4 frames per cycle.

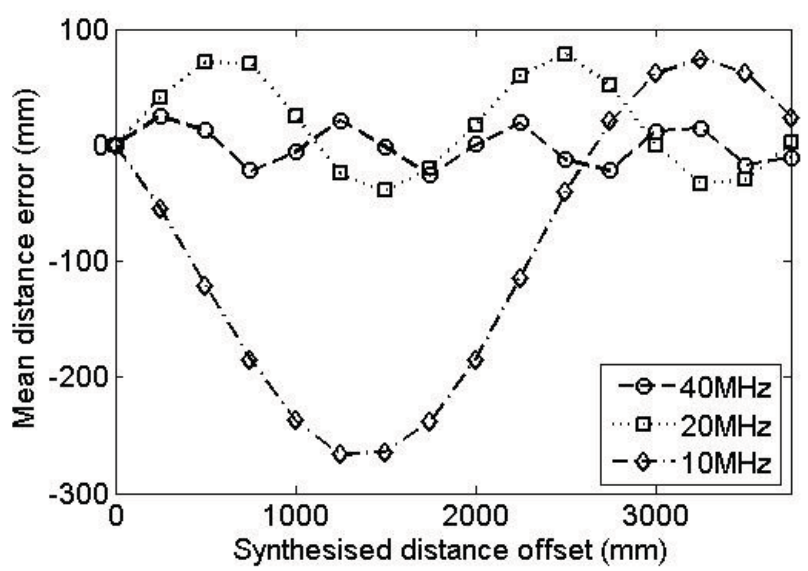

Figure 6. Mean distance measurement error versus synthesised distance offset for a $200 \mathrm{~ms}$ capture with 4 frames per cycle and modulation frequencies of 10, 20 and $40 \mathrm{MHz}$.

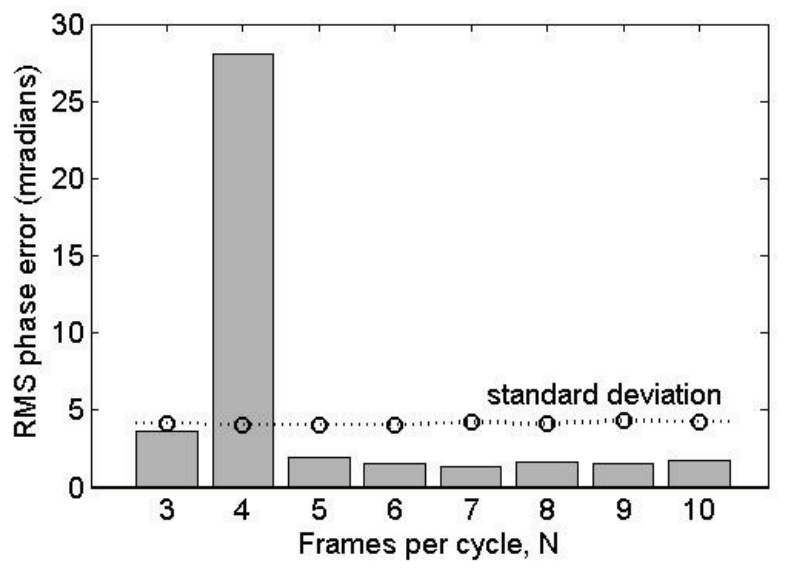

Figure 7. Bar plot of RMS phase error vs frames per cycle for $200 \mathrm{~ms}$ captures at $40 \mathrm{MHz}$ modulation. Standard deviation vs frames per cycle is shown as the dotted line. 
A correlation can be seen between the experimental results and the simulated results shown in Fig. 2, although the experimental RMS error is generally smaller. This can be attributed to a reduction in amplitude of the odd order harmonics compared to the worst case triangular waveform used for the simulation. Four frames per cycle remains approximately eight times worse than the other options, suggesting that the odd order harmonics are still significant. Three frames per cycle shows a larger RMS phase error than those greater than 4 indicating that there are likely $2^{\text {nd }}$ and $4^{\text {th }}$ order harmonics with some influence.

The dotted line across the plot shows the experimental standard deviation of the phase measurements for each $N$. The precision remains fairly constant, indicating that as $N$ increases the reduced integration time per frame is adequately compensated by the increased number of acquired frames. For all $N$ values except four the RMS phase error is below that of the system precision in this case. That is, the random errors become dominant as the systematic errors fall below the noise floor.

\section{CONCLUSION}

This paper has described the construction of a range imaging system utilising a PMDTechnologies 19k-2 image sensor and an Altera Stratix III FPGA. The especially useful aspect of this system is the ability to easily change system operating parameters through software in real-time. These include simple parameters such as the modulation frequency, the number of frames per cycle and sensor integration time, as well as more complex control over the modulation signal allowing phase, frequency and duty cycle to be changed between or within the sensor integration period.

This configurability has been utilised to characterise the system, determining phase precision and accuracy for various modulation frequencies, resulting in an optimal modulation frequency of $36 \mathrm{MHz}$.

The system has been further characterised using different frames per cycle, showing that an improvement of phase accuracy of up to ten times can be achieved by using frames per cycle other than the typical value of four.

\section{ACKNOWLEDGMENTS}

The authors would like to thank Toby Balsom for his work developing the illumination board.

\section{REFERENCES}

[1] B. Büttgen and P. Seitz, "Robust optical time-of-flight range imaging based on smart pixel structures," IEEE Trans. on Circuits and Systems I: Regular Papers, vol. 55, pp. 1512-1525, 2008.

[2] G. Gulden, D. Becker, and M. Vossiek, "Novel optical distance sensor based on MSM technology," IEEE Sensors Journal, vol. 4, no. 5, pp. 612-618, 2004.

[3] S. Kawahito et al, "A CMOS time-of-flight range image sensor with gates-on-field-oxide structure," IEEE Sensors Journal, vol. 7, no. 12, pp. 1578-1586, 2007.

[4] T. Ringbeck, "A 3D time of flight camera for object detection," Proc. Eigth Conf. Optical 3-D Measurement Techniques, 2007, http://www.pmdtec.com.

[5] G. Yahav, G. Iddan, D. Mandelbaum, “3D imaging camera for gaming application," Int. Conf. on Consumer Electronics, pp. 1-2, 2007, http://www.3dvsystems.com.

[6] T. Oggier, B. Büttgen, F. Lustenberger, "SwissRanger SR3000 and first experiences based on miniaturized 3D-TOF cameras," Swiss Center for Electronics and Microtechnology, CSEM, IEE, Fachhochschule Rapperswil Switzerland, Technical Report, 2005, http://mesaimaging.ch.

[7] S.B. Gokturk, H. Yalcin, C. Bamji, "A Time-of-Flight Depth SensorSystem Description, Issues, and Solutions," in Proc. IEEE Workshop Real-Time 3D Sensors , 2004, http://www.canesta.com.

[8] A. D. Payne, A. A. Dorrington, M. J. Cree, and D. A. Carnegie, "Improved Linearity Using Harmonic Error Rejection in a Full-Field Range Imaging System," Proc. SPIE 3D Image Capture and Applications VII, vol. 6805, pp. 68050D, 2008.

[9] A. A. Dorrington, M. J. Cree, A. D. Payne, R. M. Conroy, D. A. Carnegie, "Achieving sub-millimetre precision with a solid-state fullfield heterodyning range imaging camera," Meas. Sci. Technol., vol. 18, pp. 2809-2816, 2007.

[10] A. Jelalian, Laser Radar Systems, Artech House, Boston, 1992.

[11] A. A. Dorrington et al, "Video-rate or highprecision: a flexible range imaging camera", Image Processing: Machine Vision Applications. Proceedings of the SPIE, 6813, 681307, 2008.

[12] Altera Corporation, Stratix III Device Handbook, v1.7 edition, 2008, http://www.altera.com.

[13] PMD Technologies GmbH, Photonics PMD 19K-2 High Resolution 3D Video Sensor Array 160x120 Pixels Datasheet, Revision 1.0, 2008, http://www.pmdtec.com. 